Portland State University

PDXScholar

8-1-1995

\title{
Monte Carlo Simulations of the Extinction Rate of Densely Packed Spheres with Clustered and Nonclustered Geometries
}

\author{
Lisa M. Zurk \\ Portland State University \\ L. Tsang \\ University of Washington - Seattle Campus \\ K. H. Ding \\ Massachusetts Institute of Technology \\ Dale P. Winebrenner \\ University of Washington - Seattle Campus
}

Follow this and additional works at: https://pdxscholar.library.pdx.edu/ece_fac

Part of the Electrical and Computer Engineering Commons

Let us know how access to this document benefits you.

\section{Citation Details}

Zurk, L. M., Tsang, L., Ding, K. H., and Winebrenner, D. P. (1995). Monte Carlo simulations of the extinction rate of densely packed spheres with clustered and nonclustered geometries. JOSA A, 12(8), 1772-1781.

This Article is brought to you for free and open access. It has been accepted for inclusion in Electrical and Computer Engineering Faculty Publications and Presentations by an authorized administrator of PDXScholar. Please contact us if we can make this document more accessible: pdxscholar@pdx.edu. 


\title{
Monte Carlo simulations of the extinction rate of densely packed spheres with clustered and nonclustered geometries
}

\author{
L. M. Zurk \\ Applied Physics Laboratory, University of Washington, Seattle, Washington 98195 \\ L. Tsang \\ Department of Electrical Engineering, University of Washington, Seattle, Washington 98195
}

K. H. Ding

Research Laboratory of Electronics, Massachusetts Institute of Technology, Cambridge, Massachusetts 02139

\author{
D. P. Winebrenner
}

Applied Physics Laboratory, University of Washington, Seattle, Washington 98195

Received July 15, 1994; revised manuscript received March 10, 1995; accepted March 10, 1995

\begin{abstract}
Scattering and absorption coefficients are presented from Monte Carlo simulations of electromagnetic wave propagation in a volume of densely packed, random dielectric, absorptive spheres. The particles are modeled both with and without a surface adhesion that causes them to form clustered groups. Results for scatterer densities greater than a few percent by volume differ significantly from those obtained under the independentscattering assumption. The extinction rates agree well with analytic dense-medium theory. Results also show that, on account of local fields experienced by the particles, the system absorption is different from that predicted with an assumption of independent absorption. Scattering is increased when the spheres are deposited with surface adhesion that causes them to cluster and to form larger particles.
\end{abstract}

\section{INTRODUCTION}

To understand accurately the physics of electromagnetic scattering and emission in a medium that has a dense distribution of scatterers, one must utilize both an accurate model of the medium and an appropriately rigorous solution technique to the scattering equations. Discrete particle models in which spherical scatterers occupy random positions in the medium have been used as models to calculate scattering properties. ${ }^{1}$ In recent work we have investigated a sticky particle pair distribution function, ${ }^{2}$ which includes the adhesive character of the particles that causes them to clump together. This adhesive character provides a more accurate depiction of particles that exist in clusters (for example, dust or soot particles).

For wave propagation in a medium consisting of randomly distributed scatterers, the classic assumption is that of independent scattering, which states that the extinction rate is $n_{0} \sigma_{e}$, where $n_{0}$ is the number of particles per unit volume and $\sigma_{e}$ is the extinction cross section of an individual particle. ${ }^{3}$ It has been demonstrated experimentally ${ }^{4-6}$ and theoretically ${ }^{7,8}$ that the independent-scattering assumption is no longer valid in a dense medium. (A dense medium is a medium in which the particles occupy a significant fraction of volume, i.e., more than $5 \%$.) This is because the dense scatterer packing gives rise to correlated scattering between the particles, and dense-medium theory that includes these effects must be used for accurate results. An exact formulation of Maxwell equations in multiple-scattering form has been solved iteratively by Tsang et al. ${ }^{9}$ and used in Monte Carlo simulations to compute extinction rates for fractional volumes up to $25 \%$. This formulation is a rigorous, numerically exact treatment of multiple scattering of electromagnetic waves in a system of densely packed spheres, and it agrees with results from carefully controlled laboratory experiments of dense media. ${ }^{10}$ The number of spheres, $N$, that can be considered in these Monte Carlo simulations depends only on the available computer memory. For the iterative formulation there is an $O(N)^{2}$ memory dependence. Thus the Monte Carlo simulations provide a method of exact calculation of wave properties for large numbers of spheres. In this paper we present extinction rates calculated from systems of up to 3500 spheres and fractional volumes up to $40 \%$, for both clustered and nonclustered geometries. Simulations for systems with a smaller number of random, densely distributed spheres have been solved by Wang and Chew, ${ }^{11}$ using a recursive aggregate $T$-matrix algorithm.

Propagation and scattering in a dense medium have also been studied analytically with the effective-media approximation, ${ }^{12,13}$ the quasi-crystalline approximation ${ }^{14,15}$ (QCA), and the quasi-crystalline approximation with coherent potential ${ }^{14,16}$ (QCA-CP). These theories rely on a 
statistical description of the particle positions called the pair distribution function, which expresses the nonpenetrable nature of the particles and the constraints on their position in a densely packed system. In a recent paper ${ }^{17}$ the QCA was shown to be in good agreement with results from optical scattering in dense media. In Ref. 2 we presented extinction rates calculated with the QCA using a sticky particle pair function that shows that clustering is an important factor in scattering behavior. In this paper we show that extinction rates calculated in Monte Carlo simulations of clustered particles are also higher than those calculated for nonclustered particles.

We use Monte Carlo simulations to calculate the absorption coefficients of lossy media and to illustrate absorption enhancement. Absorption enhancement is seen in highly dense systems and consists of a different absorption rate from that which would be seen under an independent-absorption assumption. The near-field effects experienced by the particles on account of their close packing can, in some cases, enable them to absorb a greater amount of energy from the incident wave.

\section{PARTICLE PLACEMENT}

\section{A. Sticky Particles}

To model more accurately systems in which nonpenetrable particles exhibit an adhesive attraction, we utilize a sticky hard sphere (SHS) pair distribution function. In this model spherical particles of diameter $d$ demonstrate an attractive interparticle potential $u(r)$, given by ${ }^{18}$

$$
u(r)= \begin{cases}\infty & 0<r<s \\ \ln \left[\frac{12 \tau(d-s)}{d}\right] & s<r<d, \\ 0 & r<d\end{cases}
$$

where $\tau$ is inversely proportional to the strength of the attractive force, $s$ is an intermediate distance, and $d-$ $s$ is allowed to become infinitesimally small. The SHS pair distribution function $g(r)$ measures the probability of finding a particle at $r$ given a particle at $r=0$. A closed-form solution for the integral of $g(r)-1$ can be found under the Percus-Yevick approximation ${ }^{19,20}$ and is in a form that can be used in the QCA and the QCA-CP.

If one is to perform Monte Carlo simulations of systems of clustered particles described by the SHS pair distribution function $g(r)$, deposition of the particles must be influenced by the attractive square-well potential. Algorithms for shuffling until the spheres achieve a distribution given by $g(r)$ appear in papers of Seaton and Glandt $^{21}$ and Kranendonk and Frenkel. ${ }^{22}$ However, because the amount of shuffling required to achieve random placement of the particles while still satisfying the SHS model is very high, we adapted the algorithms so that the particles can be directly deposited into the test volume with the SHS potential. The deposition algorithm can be stated as follows:

(1) Calculate an effective subvolume for each particle energy state (PES) for the particle to be deposited.

(2) Determine a position for the particle that satisfies the PES.
(3) Accept or reject the position based on particle overlap.

(4) Register the new bond configuration of the system.

In molecular systems the PES of a particle corresponds to its coordination number (or number of bonds with other particles). In three-dimensional systems the highest PES attainable is 12 , which corresponds to the closest packing of neighbors. Following Seaton and Glandt, ${ }^{21}$ we have allowed moves up to PES 3 , which has been shown to be a good approximation. The calculation of subvolumes for the PES's involves integrating over the sites available for each PES, neglecting overlap. For example, the subvolume associated with zero bonds (PES 0) is just the test volume. To calculate the subvolume available for one bond, we begin by considering each sphere already deposited in the test volume. Since a single bond can occur when the test particle is placed anywhere along the surface of these spheres, the available subvolume is found by integrating over the surface of the spheres. Likewise, the subvolume for the two bonds (PES 2) is found by integrating along the circle that lies a distance $d$ from two other sphere centers. The calculation of the subvolumes involves registering the new particle configuration in the test volume after each deposition. The normalized subvolumes for the three PES's can be expressed $\operatorname{as}^{21,22}$

$$
\begin{aligned}
V^{0} & =V \\
V^{1} & =N \frac{4 \pi d^{3}}{12 \tau}, \\
V^{2} & =\sum_{i, j} \frac{\pi d^{2}}{72 \tau^{2}} r_{\mathrm{cir}}, \quad r_{i j}<2 d,
\end{aligned}
$$

where $V$ is the test volume, $N$ is the number of particles, $r_{i j}$ is the distance between the $i$ th and $j$ th particles, and $r_{\text {cir }}$ is the radius of the circle such that

$$
r_{\mathrm{cir}}=d\left[1-\left(\frac{r_{i j}}{2 d}\right)^{2}\right]^{1 / 2}
$$

The calculation of PES's requires ongoing maintenance of a catalog of the system configuration so that the number of deposited particles, $N$, and the number of particle pairs able to bond with a third the summation in Eq. (4)] are known. This catalog also contains information on the positions available in each PES. Once a PES is calculated, a position in which that PES is achieved is randomly chosen from the available sites, and the check for particle overlap is made. As expected, the number of particle collisions increases as more particles are deposited in the test volume. For higher fractional volumes (i.e., $>35 \%$ ) deposition becomes impractical, and a shuffling algorithm is necessary instead.

The pair distribution function $g(r)$ from systems of $N=2000$ particles and averaged over 30 realizations is shown in Fig. 1. Fractional volumes of $f_{v}=0.1$ and $f_{v}=0.2$, with a stickiness of $\tau=0.2$, are shown. The vertical axis represents the normalized probability, and the horizontal axis is the particle separation normalized to the particle diameter. Also shown in the figure as a 


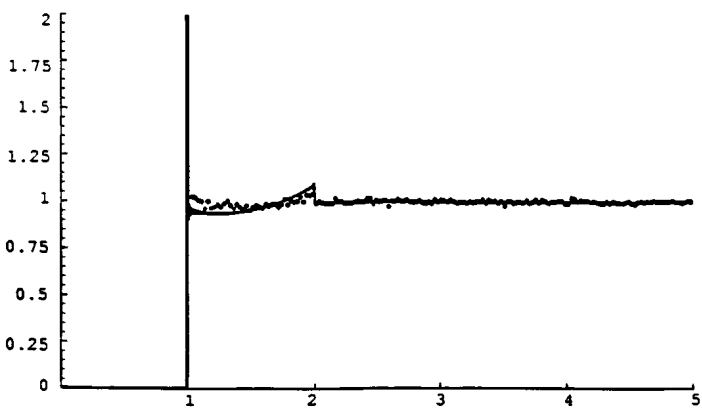

(a)

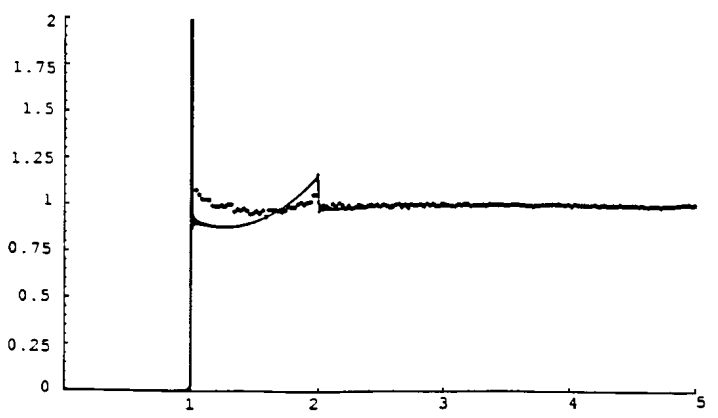

(b)

Fig. 1. Pair distribution function calculated from Monte Carlo simulations and a Percus-Yevick SHS with $\tau=0.2$ : (a) $f_{v}=$ 0.1, (b) $f_{v}=0.2$. The $x$ axis is $r / d$, and the $y$ axis is normalized probability.

solid curve is the Percus-Yevick SHS calculated as in Ref. 2, which agrees with the simulation results (agreement improves as $N$ and the number of realizations, $N_{r}$, increase). For separations $r / d<1.0, g(r)$ is zero, since there is zero probability that impenetrable particles can be separated by a distance of less than their diameter. Note the discontinuity at $r / d=2.0$, which corresponds to the inequality in Eq. (4). Physically, this represents the geometrical effect that two particles separated by a distance greater than their diameter cannot both bond with a third particle. The presence of this discontinuity is unique to the SHS pair distribution function and is representative of the clustering of the system.

A computer-generated view of a subvolume of sticky particles with $\tau=0.2$ and $f_{v}=0.35$ is shown in Fig. 2 . Among the more striking features of the clusters are their irregular and chainlike structures. In Ref. 2 we demonstrated the inability to model the scattering behavior of these clusters with spheres of a single effective size. One can obtain a description of the clusters resulting from SHS deposit by considering the fractal nature of the resulting particle groups. For a given cluster of particles we can define the radius of gyration, $R_{g},{ }^{23}$ as

$$
R_{g}=\left(\frac{1}{N_{c}} \sum_{i=1}^{N_{c}} r_{i}^{2}\right)^{1 / 2},
$$

where $N_{c}$ is the number of particles in a cluster and $r_{i}$ is the distance of the $i$ th cluster particle from the center of mass. Then there exists a logarithmic relationship between $R_{g}$ and $N_{c}$, or

$$
N_{c}=c\left(R_{g} / a\right)^{D_{f}}
$$

where $c$ is a constant and $D_{f}$ is the fractal, or Hausdorff, dimension ${ }^{24}$ of the system. The fractal nature of the sticky particle clusters provides another quantitative method by which to characterize the random media. Investigations have been made on scattering from a single fractal cluster ${ }^{25,26}$ and have shown that there is an increase in the scattering and absorption cross section as a result of the high local fields. In this paper we investigate whether this local enhancement alters the overall scattering properties when a large number of randomly placed sticky particle clusters are considered.

\section{B. Placement for Higher Fractional Volumes}

For fractional volumes greater than $35 \%$ it is impossible to create an aggregation of spheres with the deposition method. This is because the loss of freedom that is due to the higher sphere packing increases the chances of sphere collision during deposit. For high fractional volumes it is necessary instead to deposit the spheres in a uniform lattice into the test volume and then shuffle them in some fashion until a random ensemble results.

For the nonsticky spheres shuffling occurs over $N_{p}$ passes in which an attempt is made to move each sphere from its initial location to a new random location. For each sphere $\alpha$ the new location $r_{\alpha}^{\prime}$ is determined from its initial location $r_{\alpha}$ by

$$
\begin{aligned}
& x_{\alpha}^{\prime}=x_{\alpha}+\delta_{x}, \\
& y_{\alpha}^{\prime}=y_{\alpha}+\delta_{y}, \\
& z_{\alpha}^{\prime}=z_{\alpha}+\delta_{z},
\end{aligned}
$$

where $\delta_{x}, \delta_{y}$, and $\delta_{z}$ are random numbers with a magnitude between zero and some chosen $\epsilon$. If the position at $r_{\alpha}^{\prime}$ results in sphere overlap, the sphere is left in its

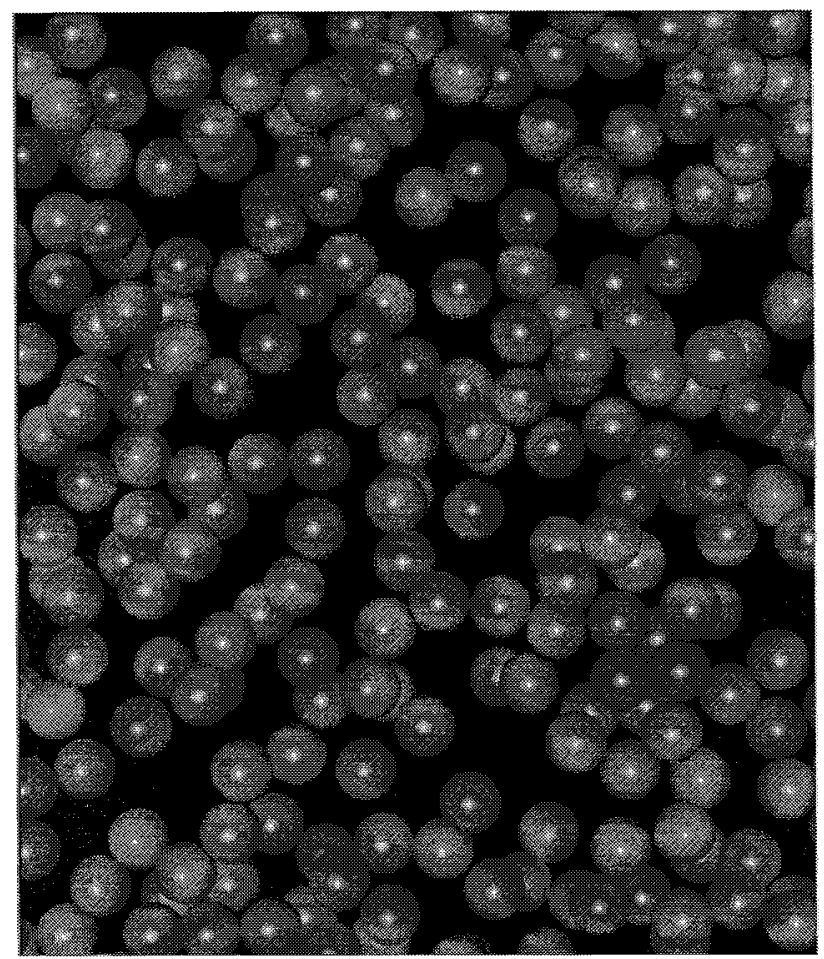

Fig. 2. Three-dimensional computer-generated view of aggregation of spheres with $f_{v}=0.35$ and $\tau=0.2$. 


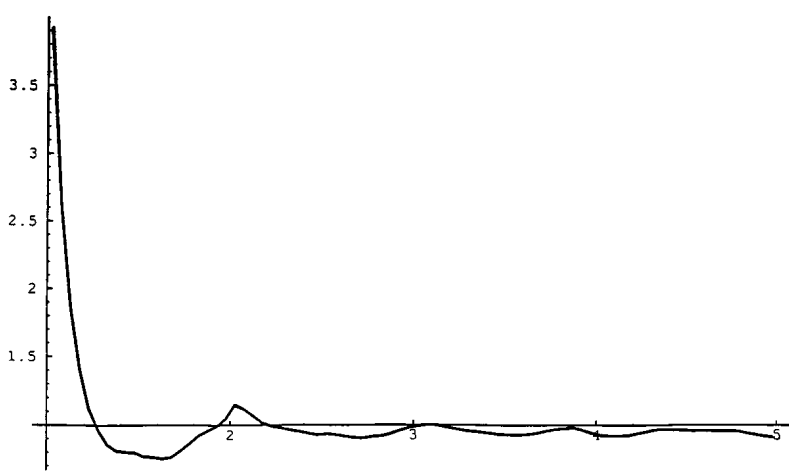

Fig. 3. Pair distribution function calculated from Monte Carlo simulations with a shuffling algorithm for a fractional volume of $40 \%$. The $y$ axis is normalized probability.

initial position and movement is attempted with the next sphere. The randomness of the final configuration depends on the number of shuffling passes, $N_{p}$, and on the movement distance $\epsilon$. If $\epsilon$ is too large, new positions will frequently collide with other spheres, and few successful moves will result. On the other hand, a very small $\epsilon$ will cause such small shifts in the sphere position that the final position will not be substantially different from the original. The optimal value of $\epsilon$ depends on the sphere packing and can be determined by trial and error. We have found that the pair distribution function of an insufficiently shuffled organization of spheres is indistinguishable from that of an adequately shuffled organization and thus is not an ideal indicator of randomness. Instead, the univariate particle statistics (i.e., the probability of finding a particle in a given location) can be used. For a truly random organization the particle position should be uniformly distributed.

Once a random organization has been achieved, subsequent realizations are constructed by shuffling with $N_{p}=200$ and $\epsilon=0.1 \Delta$, where $\Delta$ is the original lattice spacing. Figure 3 shows the pair distribution function for 3500 spheres and a fractional volume of $40 \%$, averaged over 15 realizations. The form of $g(r)$ in Fig. 3 agrees well with the Percus-Yevick hard-sphere pair distribution function.

\section{SOLUTION OF MULTIPLE-SCATTERING EQUATIONS}

To calculate the extinction and absorption coefficients of a system of densely packed spheres, we iteratively solve Maxwell equations that are cast in multiple-scattering form. The formulation is numerically exact and contains no approximations. For each realization of $N$ spheres the positions are generated randomly in the manner described in Section 2. We then solve the multiplescattering equations exactly.

Maxwell equations cast into the Foldy-Lax mutiplescattering equations can be expressed in matrix notation $\mathrm{as}^{16}$

$$
\mathbf{a}^{s(\alpha)}=\sum_{\substack{\beta=1 \\ \beta \neq \alpha}}^{N} \mathbf{T}^{\alpha} \boldsymbol{\sigma}\left(k \mathbf{r}_{\alpha} \mathbf{r}_{\beta}\right) \mathbf{a}^{s(\beta)}+\exp \left(i \mathbf{k}_{i} \cdot \mathbf{r}_{\alpha}\right) \mathbf{T}^{\alpha} \mathbf{a}_{\mathrm{inc}}
$$

where
- $\mathbf{a}^{s(\alpha)}$ is the vector of coefficients for spherical wave harmonics of the multiple-scattered field for particle $\alpha$;

- $\mathbf{a}_{\text {inc }}$ is the coefficient of the incident wave;

- $k$ is the wave number of the background media;

- $\mathbf{k}_{i}$ is the wave number of the incident wave;

- $N$ is the number of spheres in the containing volume;

- $\boldsymbol{\sigma}\left(k \mathbf{r}_{\alpha} \mathbf{r}_{\beta}\right)$ is the vector spherical wave transformation matrix;

- $\mathbf{T}^{\alpha}$ is the $T$ matrix for scatterer $\alpha$, which depends on the permittivity and the radius of $\alpha$ as well as on the background permittivity;

- $\mathbf{r}_{\alpha}$ and $\mathbf{r}_{\beta}$ are the centers of particles $\alpha$ and $\beta$, respectively.

For plane-wave excitation of a single sphere, Mie theory and the $T$-matrix method yield the same results. However, in a dense collection of spheres the field experienced (called the exciting field) by any particle is due to both the incident field and the scattered field from neighboring particles. When this exact exciting field-which is, in general, not a plane wave-is known in the near, intermediate, and far fields, it is necessary to use the $T$-matrix method. This allows for decomposition of the exciting field into vector spherical waves. The $T$ matrix then specifies the scatterer's response to any type of excitation. The correspondence between the $T$ matrix and the Mie coefficients can be seen by examination of the $T$ matrix for a spherical scatterer:

$$
\begin{aligned}
\mathbf{T} & =\left[\begin{array}{cc}
\mathbf{T}^{(11)} & \mathbf{0} \\
\mathbf{0} & \mathbf{T}^{(22)}
\end{array}\right] \\
T_{m n m^{\prime} n^{\prime}}^{(11)} & =\delta_{m m^{\prime} n n^{\prime}} T_{n}^{(M)} \\
T_{m n m^{\prime} n^{\prime}}^{(2)} & =\delta_{m m^{\prime} n n^{\prime}} T_{n}^{(N)} \\
T_{n}^{(M)} & =\frac{j_{n}\left(k_{s} a\right)\left[k a j_{n}(k a)\right]^{\prime}-j_{n}(k a)\left[k_{s} a j_{n}\left(k_{s} a\right)\right]^{\prime}}{j_{n}\left(k_{s} a\right)\left[k a h_{n}(k a)\right]^{\prime}-h_{n}(k a)\left[k_{s} a j_{n}\left(k_{s} a\right)\right]^{\prime}} \\
T_{n}^{(M)}= & \frac{k_{s}{ }^{2} a^{2} j_{n}\left(k_{s} a\right)\left[k a j_{n}(k a)\right]^{\prime}-k^{2} a^{2} j_{n}(k a)\left[k_{s} a j_{n}\left(k_{s} a\right)\right]^{\prime}}{k_{s}{ }^{2} a^{2} j_{n}\left(k_{s} a\right)\left[k a h_{n}(k a)\right]^{\prime}-k^{2} a^{2} h_{n}(k a)\left[k_{s} a j_{n}\left(k_{s} a\right)\right]^{\prime}} .
\end{aligned}
$$

Thus $T_{n}^{(M)}$ and $T_{n}^{(N)}$ are equivalent to the Mie coefficients $b_{n}$ and $a_{n},{ }^{27}$ respectively, for a plane-wave excitation.

The final scattered field $\mathbf{E}_{s}$ from $N$ spheres at an observation point $r$ is

$$
\mathbf{E}_{s}(r)=\sum_{m n} \gamma_{m n}\left[a_{m n}^{s(M)} \mathbf{M}_{m n}(k r, \theta, \phi)+a_{m n}^{s(N)} \mathbf{N}_{m n}(k r, \theta, \phi)\right],
$$

where $\mathbf{M}_{m n}$ and $\mathbf{N}_{m n}$ are outgoing vector spherical wave functions and $\gamma_{m n}$ is a normalization coefficient. ${ }^{16}$

Equation (11) can be derived directly from Maxwell equations for the case of discrete scatterers in a homogeneous background. Unlike continuous random media, discrete scatterers have well-defined boundaries. Since the space occupied by the random scatterers and the space occupied by the background are distinguishable, with each region having its own permittivity, the fields in each region can be expressed in a complete spherical wave expansion and equated at the scatterer boundaries. A derivation utilizing dyadic Green's functions is given by Peterson and Ström ${ }^{28}$ and also in Ref. 16. For an 
$N$-particle system occupying volumes $V_{1}, V_{2}, \ldots, V_{N}$, where the $j$ th particle has the wave number $k_{j}=\sqrt{\mu \epsilon_{j}}$, the $N$-particle dyadic Green's function can be written as

$$
\begin{aligned}
\mathbf{G}\left(\mathbf{r}, \mathbf{r}^{\prime}\right)= & \mathbf{G}_{0}\left(\mathbf{r}, \mathbf{r}^{\prime}\right)+\sum_{l=1}^{N} \int_{V_{l}} \mathrm{~d} r^{\prime \prime} \mathbf{G}_{0}\left(\mathbf{r}, \mathbf{r}^{\prime \prime}\right)\left(k_{l}^{2}-k^{2}\right) \\
& \times \mathbf{G}\left(\mathbf{r}^{\prime \prime}, \mathbf{r}^{\prime}\right),
\end{aligned}
$$

where $\mathbf{G}_{0}$ is the free-space Green's function and the above equation is valid for all $\mathbf{r}$ and $\mathbf{r}^{\prime}$. To put the scattering equation in more compact form, we will use Dirac's operator notation. Equation (18) in coordinate operator notation is

$$
\mathbf{G}=\mathbf{G}_{0}+\mathbf{G}_{0} \sum_{j=1}^{N} \mathbf{U}_{j} \mathbf{G},
$$

where $\mathbf{U}_{j}=U_{j} \mathbf{I}=U\left(\mathbf{r}-\mathbf{r}_{j}\right) \mathbf{I}$ and

$$
U\left(\mathbf{r}-\mathbf{r}_{j}\right)=\left\{\begin{array}{ll}
0 & \mathbf{r} \operatorname{not} \text { in } V_{j} \\
k_{j}{ }^{2}-k^{2} & \mathbf{r} \text { in } V_{j}
\end{array} .\right.
$$

Equation (19) expresses the $N$-particle Green's function in terms of the potential operator $\mathbf{U}_{j}$. Using the transition operator $\mathbf{T}_{j}=\left(\mathbf{I}-\mathbf{U}_{j} \mathbf{G}_{0}\right)^{-1} \mathbf{U}_{j}$ for particle $j$, we can write Eq. (19) as

$$
\begin{gathered}
\mathbf{G}_{j}=\mathbf{G}_{0}+\mathbf{G}_{0} \sum_{l=1, l \neq j}^{N} \mathbf{T}_{l} \mathbf{G}_{l}, \\
\mathbf{G}=\mathbf{G}_{0}+\mathbf{G}_{0} \sum_{j=1}^{N} \mathbf{T}_{j} \mathbf{G}_{j},
\end{gathered}
$$

which gives the $N$-particle Green's function in terms of the $\mathbf{T}_{j}$ operator and the $j$ th-particle Green's function $\mathbf{G}_{j}$. Equation (11) is the result when the dyadic Green's function in the background and within the particles is expanded in vector spherical wave functions, which constitute a complete basis.

An alternative method is to write the operator equations (21) and (22) in momentum representation for random distributions of scatterers. This has been discussed in Ref. 29. In Appendix A of that paper it is shown that for discrete scatterers in a homogeneous background the momentum representation of the operator equations (21) and (22) is equivalent to the $T$-matrix approach.

\section{A. Absorption Calculation}

The internal electric field $\mathbf{E}_{\text {int }}$ in each sphere can likewise be represented as

$$
\begin{aligned}
\mathbf{E}_{\mathrm{int}}(r)= & \sum_{m n} \gamma_{m n}\left[c_{m n}^{(M)} \operatorname{Rg} \mathbf{M}_{m n}(k r, \theta, \phi)\right. \\
& \left.+c_{m n}^{(N)} \operatorname{Rg} \mathbf{N}_{m n}(k r, \theta, \phi)\right]
\end{aligned}
$$

where $\operatorname{Rg} \mathbf{M}_{m n}$ and $\operatorname{Rg} \mathbf{N}_{m n}$ are the regular vector spherical wave functions. (Regular vector spherical wave functions are labeled with the prefix $\mathrm{Rg}$ to denote replacement in the wave function of the spherical Hankel function $h_{n}$ by the spherical Bessel function $j_{n} \cdot{ }^{16}$ ) The relationship between the scattered field coefficients $a_{m n}^{s}$ and the internal field coefficients $c_{m n}$ is given by ${ }^{16}$

$$
\mathbf{c}=\left(-\operatorname{Rg} \mathbf{Q}^{t}\right)^{-1} \mathbf{a}^{s} .
$$

The matrix $\operatorname{Rg} \mathbf{Q}^{t}$ can be determined by equating the tangential fields at the particle's surface and is diagonal for spheres:

$$
R_{g} \mathbf{Q}^{t}=\left[\begin{array}{cc}
R_{g} \mathbf{P} & \mathbf{0} \\
\mathbf{0} & R_{g} \mathbf{U}
\end{array}\right]
$$

with

$$
\begin{aligned}
R_{g} P_{m n m^{\prime} n^{\prime}} & =-i k k_{s} J_{m n m^{\prime} n^{\prime}}^{(21)}-i k^{2} J_{m n m^{\prime} n^{\prime}}^{(12)}, \\
R_{g} U_{m n m^{\prime} n^{\prime}} & =-i k k_{s} J_{m n m^{\prime} n^{\prime}}^{(12)}-i k^{2} J_{m n m^{\prime} n^{\prime}}^{(21)}, \\
J_{m n m^{\prime} n^{\prime}}^{(12)} & =a^{2} \delta_{m m^{\prime}} \delta_{n n^{\prime}} j_{n}\left(k_{s} a\right) \frac{\left[k a j_{n}(k a)\right]^{\prime}}{k a}, \\
J_{m n m^{\prime} n^{\prime}}^{(21)} & =-a^{2} \delta_{m m^{\prime}} \delta_{n n^{\prime}} j_{n}(k a) \frac{\left[k_{s} a j_{n}\left(k_{s} a\right)\right]^{\prime}}{k_{s} a},
\end{aligned}
$$

where $j_{n}$ is the spherical Bessel function and $k_{s}$ is the wave number in the scatterers. Substituting the expression for $R_{g} \mathbf{Q}^{t}$ into Eq. (24) gives

$$
\begin{aligned}
c_{m n}^{M}= & \left\{i k a \left(j_{n}(k a)\left[k_{s} a j_{n}\left(k_{s} a\right)\right]^{\prime}\right.\right. \\
& \left.\left.-j_{n}\left(k_{s} a\right)\left[k a j_{n}(k a)\right]^{\prime}\right)\right\}^{-1} a_{m n}^{M}, \\
c_{m n}^{N}= & \left(i a \left\{\frac{k^{2}}{k_{s}} j_{n}(k a)\left[k_{s} a j_{n}\left(k_{s} a\right)\right]^{\prime}\right.\right. \\
& \left.\left.-k_{s} j_{n}\left(k_{s} a\right)\left[k a j_{n}(k a)\right]^{\prime}\right\}\right)^{-1} a_{m n}^{N},
\end{aligned}
$$

where the prime indicates differentiation with respect to the Bessel function argument. The internal field in Eq. (23), together with Eqs. (30) and (31), can then be used to calculate the system absorption cross section given by

$$
\sigma_{a N}=\sum_{\beta=1}^{N} \int_{V} k_{s} \epsilon_{r}^{\prime \prime}\left|\mathbf{E}_{\text {int }}^{\beta}\left(r^{\prime}\right)\right|^{2} \mathrm{~d} V^{\prime},
$$

where the integration is over the sphere volume $V$ and $\epsilon_{r}^{\prime \prime}$ is the imaginary part of the relative permittivity for the spheres. Because of orthogonality of the spherical wave functions, the expression for $\sigma_{a N}$ involves no coupling between the electric and magnetic dipole contributions (i.e., with coefficients $c_{m n}^{M}$ and $c_{m n}^{N}$ for $n=1$ ), and contributions from these two fields can be considered separately. The absorption rate $\kappa_{a}$ for a volume $V$ is given by $\kappa_{a}=\sigma_{a N} / V$.

The absorption under the independent assumption can be expressed in terms of the Mie absorption cross section $\sigma_{a}$, where $\sigma_{a}=\sigma_{t}-\sigma_{s}$ and

$$
\begin{aligned}
\sigma_{t} & =\frac{2 \pi}{k^{2}} \sum_{n=1}^{\infty}(2 n+1)\left[\operatorname{Re}\left(a_{n}+b_{n}\right)\right], \\
\sigma_{s} & =\frac{2 \pi}{k^{2}} \sum_{n=1}^{\infty}(2 n+1)\left(\left|a_{n}\right|^{2}+\left|b_{n}\right|^{2}\right),
\end{aligned}
$$

where $\sigma_{t}$ and $\sigma_{s}$ are the Mie total and scattering cross sections, respectively, and $a_{n}$ and $b_{n}$ are the Mie field coefficients. The absorption coefficient for $N$ spheres in a volume $V$ is given by $\kappa_{a}=N \sigma_{a} / V$. 
In dense media the scattered field from a particle is a result of both the incident field and the fields from surrounding particles, as expressed in Eq. (11). The Mie absorption cross section in Eq. (11) assumes that the absorption is due to the incident plane wave. Thus at higher fractional volumes, where the close sphere packing greatly influences the local exciting field that a given particle experiences, the internal field can be quite different from that predicted by independent Mie calculation.

\section{B. Scattering Calculation}

In the Monte Carlo simulations aggregations of spheres are randomly placed within $N_{r}$ test volumes in a manner corresponding to either a sticky or a nonsticky pair distribution function $g(r)$. The scattered fields are calculated by use of Eqs. (11) and (17) and averaged over $N_{r}$ realizations. The averaged field can be decomposed into a coherent and an incoherent part, where the extinction rate arises from the incoherent scattered field. The coherent scattered field $\left\langle E_{s}\right\rangle$ is calculated by

$$
\left\langle\mathbf{E}_{s}\right\rangle=\frac{R}{N_{r}} \sum_{\sigma=1}^{N_{r}} \mathbf{E}_{s}^{\sigma}
$$

and the incoherent field is $\mathcal{F}_{s}^{\sigma}=\mathbf{E}_{s}^{\sigma}-\left\langle\mathbf{E}_{s}\right\rangle$, where $\sigma$ is the realization index with $\sigma=1,2, \ldots, N_{r}$ realizations. Calculations of the scattering coefficient $\kappa_{s}$ from the incoherent scattered field $\boldsymbol{F}_{s}$ can be expressed as

$$
\kappa_{s}=\frac{1}{V} \int_{0}^{\pi} \mathrm{d} \theta_{s}\left(\sin \theta_{s}\right) \int_{0}^{2 \pi} \mathrm{d} \phi_{s} \frac{R^{2}}{N^{r}} \sum_{\sigma=1}^{N_{r}}\left|\mathcal{E}_{s}\right|^{2} .
$$

\section{Choice of Statistical Sample}

The Monte Carlo volume must satisfy three criteria. First, the test volume must be small enough that $V \ll$ $\left(1 / \kappa_{e}\right)^{3}$; then the attenuation of the original incident wave traveling through the volume is negligible. The volume is not, however, infinitesimally small but is large in comparison with the wavelength $\left(V \gg \lambda^{3}\right)$, so that, second, the phase of the wave varies appreciably across the volume to create random phase situations. Last, the number of enclosed spheres in the volume must be large enough to represent a random sampling of scatterers $(N \gg 1)$. When the scattered fields are calculated within such a volume, the randomness of the wave's phase and of the particle's position destroys some of the phase coherence in the system and serves to speed the convergence of the iteration.

\section{Computational Consideration}

The solution to Eq. (11) can be obtained through iteration. The result for the $(\nu+1)$ th iteration is

$$
\mathbf{a}^{s(\alpha)(\nu+1)}=\sum_{\substack{\beta=1 \\ \beta \neq \alpha}}^{N} \mathbf{T}^{\alpha} \boldsymbol{\sigma}\left(k \mathbf{r}_{\alpha} \mathbf{r}_{\beta}\right) \mathbf{a}^{s(\beta)(\nu)}+\exp \left(i \mathbf{k}_{i} \cdot \mathbf{r}_{\alpha}\right) \mathbf{T}^{\alpha} \mathbf{a}_{\mathrm{inc}},
$$

where the superscript $\nu$ denotes the $\nu$ th-iterated solution and the initial solution is just the incident field coefficients. To obtain the final solution for $\mathbf{a}^{s(\alpha)(\nu+1)}$, we iterate the system of equations (37) until the maximum change in the field coefficients from one iteration to the next is less than $5 \%$, at which point the solution is considered to have converged.

As stated in Subsection 3.C, the random sphere distribution increases the speed of convergence. This effect was observed in our initial attempts to solve Eq. (37) for a system with random, nonsticky scatterers occupying $40 \%$ by volume. As described in Subsection 2.B, a system this dense requires initial periodic particle placement and then sufficient shuffling, so that the resulting positions are random. In Fig. 4 we show the number of iterations necessary for convergence of Eq. (37) when inadequate shuffling causes some remaining periodicity in the sphere placement. Figure 4 shows the number of realizations along the horizontal axis (where each realization results from shuffling the previous one, and thus randomness increases from left to right). The effect of even a slight amount of periodicity is an increase in the number of iterations necessary for convergence, because of the destruction of the system's random nature. Thus the first few realizations in this attempt required more than 50 iterations, compared with $\sim 30$ when the periodicity was decreased by additional shuffles. For $f_{v}=40 \%$ and $k a=0.2$ a truly random configuration required 23 iterations for the results to converge.

The number of iterations necessary for convergence depends on the fractional volume of the spheres, the dielectric contrast, and the sphere radius. In this paper we present results for spheres with permittivity $\epsilon_{s}=3.2 \epsilon_{0}$ and with size parameter $k_{s} a=0.2$. Thus the number of iterations depends on the fractional volume and the particle position. For the lower-density case $f_{v}=15 \%$ approximately seven iterations were necessary for the solution to converge, in contrast with the 23 iterations for $f_{v}=40 \%$. This makes sense physically, as it indicates that multiple-scattering effects become more important for highly dense systems. When the sphere size is small, the $T$ matrix becomes small and the number of iterations decreases. For the case $k a=0.1$ the number of iterations needed for convergence remains fixed at 6 for all fractional volumes.

The dimension of $\mathbf{a}^{s(\alpha)}$ is determined by the number of spherical harmonics that are considered. For sparse systems with small scatterers it is sufficient to consider

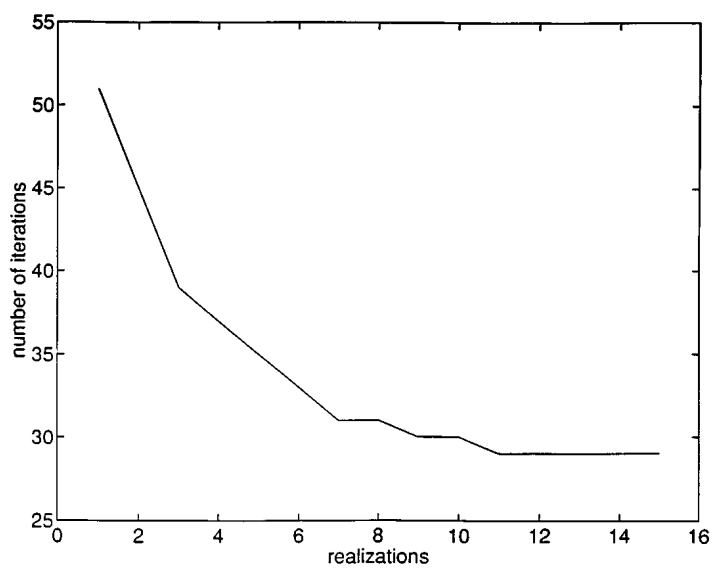

Fig. 4. Number of iterations versus realizations for 3500 spheres that are $40 \%$ by volume with $k a=0.2$. The initial realization has some periodicity that is destroyed by shuffling in later realizations, and thus randomness increases from left to right. 
only the electric dipole term. Once the particles become more closely packed, the near-field effects become important and higher-order multipoles need to be considered. In this paper we calculate the field that is due to both the electric and the magnetic dipoles. In this formulation $\mathbf{a}^{s(\alpha)}$ is a $6 \times 1$ column vector and $\left.\boldsymbol{\sigma}\left(k \mathbf{r}_{\alpha} \mathbf{r}_{\beta}\right)\right)$ is a $6 \times 6$ translation matrix. Thus the matrix in Eq. (11) has rank $6 N$ (e.g., 18,000 for a volume containing 3000 spheres). Because this exceeds the available memory capacity, we calculate each matrix element and the spherical translation matrix as they are needed and discard them after each use. The computation time for a single iteration is thus $O\left[(M N)^{2}\right]$, where $M$ is the number of spherical harmonics considered, which is $M=6$ in the dipole case. As stated above, the number of iterations needed for even the most dense aggregation is small compared with $N$, and the overall computational time is $O\left[(M N)^{2}\right]$ for the iterative solution. Of course, Monte Carlo simulations require averaging over $N_{r}$ realizations.

\section{RESULTS}

In this section we present results from the Monte Carlo simulations of aggregations of spheres. We consider sticky and nonsticky particles and particles with a nonzero loss tangent. All the Monte Carlo calculations include both the electric and magnetic dipole contributions unless specified otherwise.

\section{A. Sticky and Nonsticky Particles}

We performed Monte Carlo simulations of nonsticky, lossless particles with size factors of $k a=0.2$ and $k a=0.1$, permittivity of $\epsilon_{s}=3.2 \epsilon_{0}$, and random organization for fractional volumes of $15 \%, 25 \%, 35 \%$, and $40 \%$. At $40 \%$ fractional volume the sphere packing made sequential deposition impossible, and we used the shuffle algorithm outlined in Subsection 2.B. Tables 1 and 2 give the simulation parameters and the computed extinction rates, which are also shown graphically in Fig. 5 along with extinction rates calculated by use of the independentscattering assumption, the QCA, and the QCA-CP. The figure shows the overestimation of the extinction rates when the independent-scattering assumption is made.
The curves for the $\mathrm{QCA}$ and the $\mathrm{QCA}-\mathrm{CP}$ increase as a function of fractional volume to a maximum of approximately $15 \%$ and then begin again to decrease, with the QCA underpredicting the scattering. The fractional volume at which maximal attenuation occurs depends on the size parameter of the particles. The Monte Carlo results (shown as asterisks) agree fairly well with those obtained with the QCA-CP, until the higher fractional volumes are reached, at which point the Monte Carlo simulations predict greater attenuation than does the QCA-CP. Initially we thought that this difference could be due to the effect of the magnetic dipole, which is included in the Monte Carlo scattering calculations but is not present in the QCA-CP solution. However, Monte Carlo simulations containing only the electric dipole produced attenuations that were only slightly lower than those from simulations with both the electric and magnetic dipoles. It seems that as the sphere packing becomes closer in the higher fractional volumes, the near fields generated by neighboring particles produce effects on the spheres that are not included in the low-frequency approximations used in the QCA-CP. The validity of the lowfrequency approximation thus appears to be dependent on the density of the scatterers.

One can see this by comparing the results in Fig. 5(a), which are for $k a=0.2$, with those in Fig. 5(b), which are for particles with $k a=0.1$. At $35 \%$ fractional volume there is a difference between the attenuation predicted by the QCA-CP and by the Monte Carlo simulations for the case $k a=0.2$ but not for $k a=0.1$. At $40 \%$ fractional volume results from particles of both sizes show a difference between the Monte Carlo simulations and the QCA-CP, but it is less pronounced for the smaller spheres. As the density of the system is increased, the particle size must be decreased to satisfy the low-frequency solution to the QCA-CP.

In Fig. 6 the Monte Carlo results are shown for spheres with $k a=0.2$ and $\epsilon_{s}=3.2 \epsilon_{0}$ for spheres both with and without an adhesive potential. The spheres with an adhesive potential were deposited as discussed in Section 2 with a stickiness of $\tau=0.2$ and a pair distribution function $g(r)$ as shown in Fig. 1. From Fig. 6 it can be clearly seen that the sticky particles (asterisks) show a greater

Table 1. Numerical Results for Lossless Spheres with $k a=0.2$ and $\epsilon_{s}=3.2 \epsilon_{0}$

\begin{tabular}{|c|c|c|c|c|c|c|c|}
\hline \multirow{2}{*}{$\begin{array}{l}\text { Fractional } \\
\text { Volume }\end{array}$} & \multicolumn{4}{|c|}{ Normalized Extinction Rate $\kappa_{e} / k\left(\times 10^{-4}\right)$} & \multirow{2}{*}{$\begin{array}{c}\text { No. of Spheres } \\
N \\
\end{array}$} & \multirow{2}{*}{$\begin{array}{c}\text { No. of Iterations } \\
N_{I} \\
\end{array}$} & \multirow{2}{*}{$\begin{array}{l}\text { No. of Realizations } \\
N_{r}\end{array}$} \\
\hline & Independent & $\mathrm{QCA}$ & $\mathrm{QCA}-\mathrm{CP}$ & Monte Carlo & & & \\
\hline $15 \%$ & 4.28 & 1.38 & 1.80 & 1.61 & 3000 & 7 & 15 \\
\hline $25 \%$ & 7.15 & 1.08 & 1.60 & 1.48 & 3000 & 9 & 15 \\
\hline $35 \%$ & 10.00 & 0.69 & 1.12 & 1.60 & 3000 & 15 & 15 \\
\hline $40 \%$ & 11.45 & 0.52 & 0.88 & 1.67 & 3500 & 23 & 20 \\
\hline
\end{tabular}

Table 2. Numerical Results for Lossless Spheres with $k a=0.1$ and $\epsilon_{s}=3.2 \epsilon_{0}$

\begin{tabular}{|c|c|c|c|c|c|c|c|}
\hline \multirow{2}{*}{$\begin{array}{c}\text { Fractional } \\
\text { Volume }\end{array}$} & \multicolumn{4}{|c|}{ Normalized Extinction Rate $\kappa_{e} / k\left(\times 10^{-5}\right)$} & \multirow{2}{*}{$\begin{array}{c}\text { No. of Spheres } \\
N\end{array}$} & \multirow{2}{*}{$\begin{array}{c}\text { No. of Iterations } \\
N_{I}\end{array}$} & \multirow{2}{*}{$\begin{array}{c}\text { No. of Realizations } \\
\qquad N_{r}\end{array}$} \\
\hline & Independent & QCA & QCA-CP & Monte Carlo & & & \\
\hline $15 \%$ & 5.37 & 1.72 & 2.25 & 1.90 & 3000 & 6 & 15 \\
\hline $25 \%$ & 8.95 & 1.35 & 2.00 & 1.50 & 3000 & 6 & 15 \\
\hline $35 \%$ & 12.53 & 0.86 & 1.40 & 1.35 & 3000 & 6 & 15 \\
\hline $40 \%$ & 14.32 & 0.65 & 1.10 & 1.63 & 3500 & 6 & 20 \\
\hline
\end{tabular}




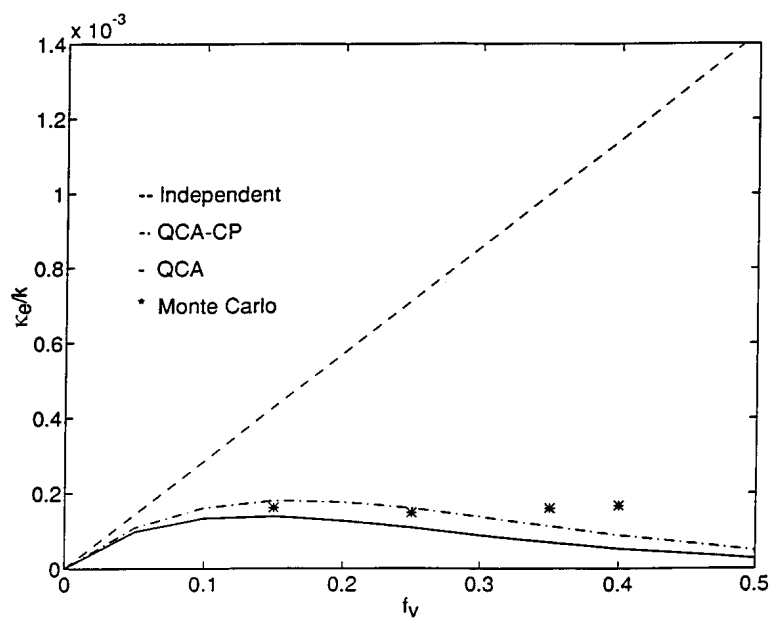

(a)

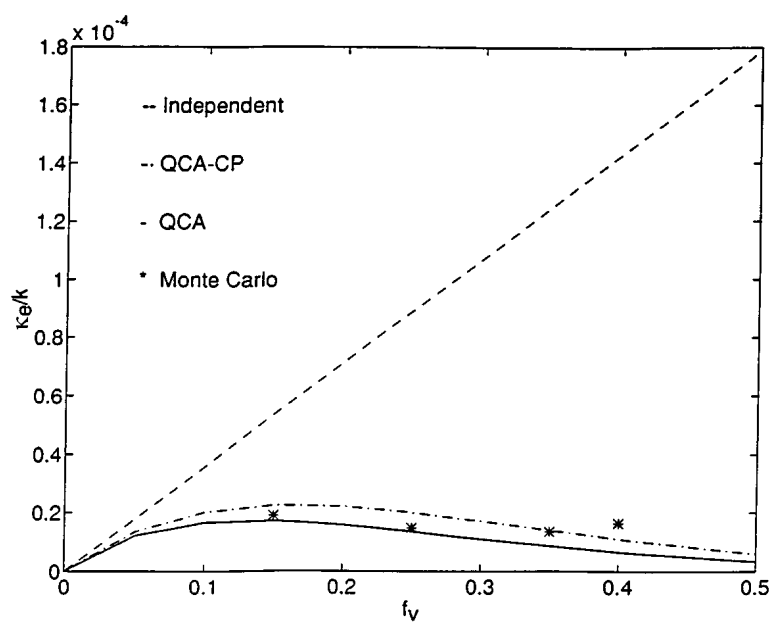

(b)

Fig. 5. Extinction rate as a function of fractional volume; the calculations are based on independent-assumption, QCA, QCA-CP, and Monte Carlo simulations. (a) $k a=0.2$, (b) $k a=0.1$.

extinction rate than do the nonsticky particles (open circles). Since the only difference between the two simulations is the pair distribution function, we can draw the important conclusion that the particle placement is a crucial factor in estimating the extinction properties. This is intuitive when the placement is influenced by a clustering nature, and the particles clump to effectively form larger particles. As one would expect, these larger particles exhibit a greater amount of attenuation because of their size. However, as we showed in Ref. 2, modeling the irregular clusters as large spheres produces incorrect results. Again, intuitively, one would expect different scattering characteristics from a chainlike structure with a high axial ratio than from a sphere of comparable size. In particular, the local polarization of the clusters will differ greatly. Even though the orientation of the clusters of spheres is random, the behavior of the wave does respond to the irregularities of these structures.

In Ref. 2 we presented extinction rates for sticky spheres calculated with the QCA and the QCA-CP. Also shown in Fig. 6 with the results from Monte Carlo simulations are results calculated with the QCA-CP. Both calculation methods yield extinction rates that are greater than those calculated from nonsticky spheres. However, the QCA-CP results are higher for a given $\tau$ than the Monte Carlo results.

\section{B. Lossy Particles}

As described in Subsection 3.A, the absorption rate of densely packed spheres can differ from that given by the independent assumption. This effect of enhanced absorption is shown in Fig. 7. The figure shows the absorption, scattering, and extinction coefficients of $N=2000$ spheres with a permittivity of $\epsilon_{s}=(3.2+i 0.01) \epsilon_{0}$ and a size factor of $k a=0.2$ as a function of fractional volume. The absorption rates were calculated from Monte Carlo simulations of randomly deposited spheres (asterisks) and from the independent Mie absorption assumption for a system of $N$ spheres (dotted curve). The absorption rate when the internal field is calculated explicitly in the Monte Carlo simulations is higher than that predicted from the independent assumption (approximately $25 \%$ greater at $30 \%$ fractional volume).

The calculated absorption rate for the higher fractional volumes is also greater than that predicted with the Maxwell-Garnett mixing formula ${ }^{30}$ (MG):

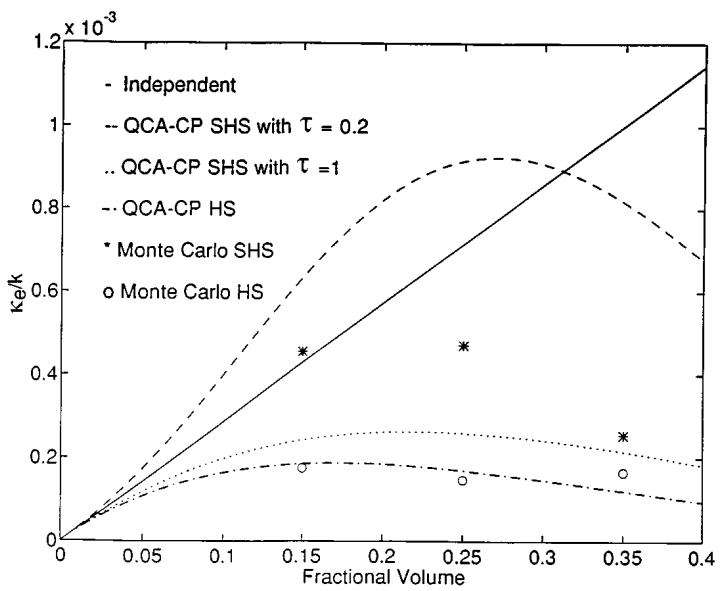

Fig. 6. Extinction rate as a function of fractional volume for nonsticky spheres and sticky spheres with $\tau=0.2$ and $\tau=1.0$ and for $k a=0.2$; the calculations are based on independent-assumption, QCA-CP, and Monte Carlo simulations.

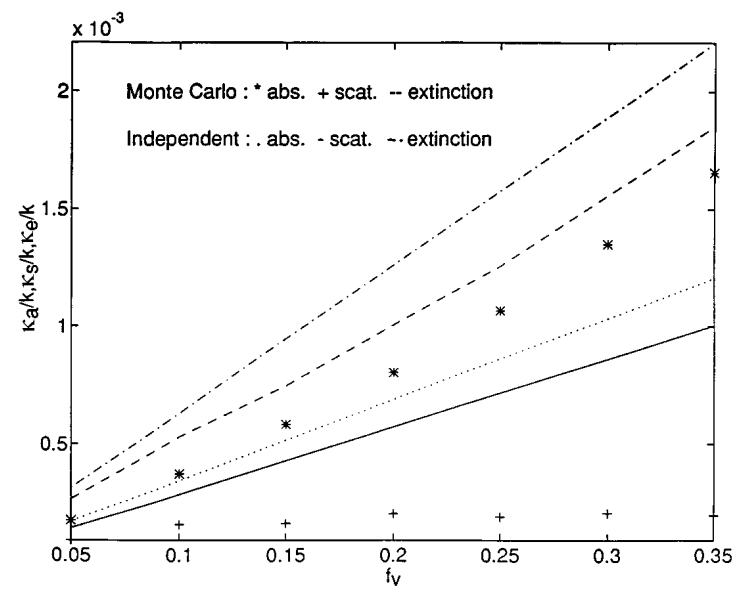

Fig. 7. Absorption rate as a function of fractional volume from Monte Carlo simulations and independent Mie absorption for spheres with $\epsilon_{s}=(3.2+i 0.01) \epsilon_{0}$ and $k a=0.2$. 


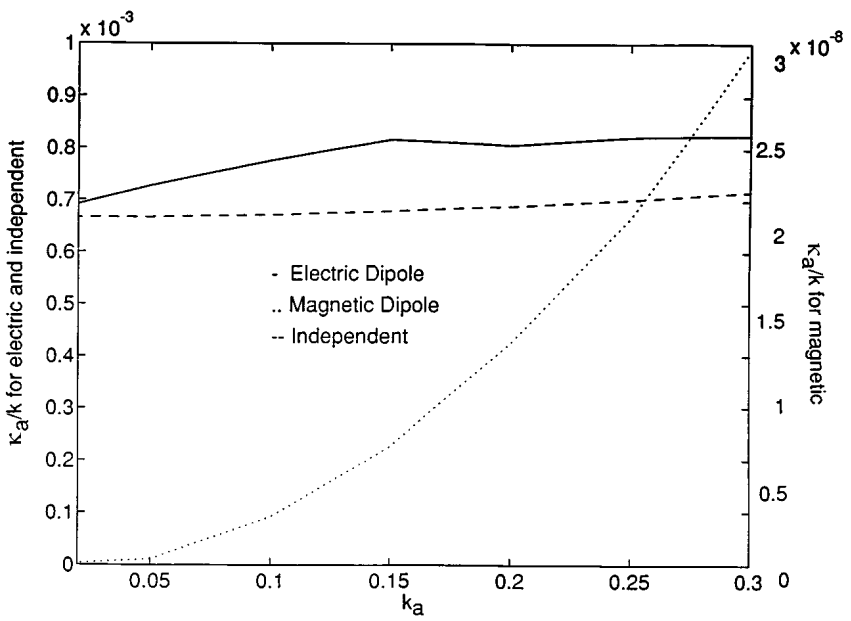

Fig. 8. Absorption rate as a function of size $k a$ from Monte Carlo simulations of spheres with $f_{v}=0.2$ for electric dipole and magnetic dipole.

$$
\begin{aligned}
\kappa_{a} / k & =\epsilon_{\mathrm{eff}}^{\prime \prime} / \sqrt{\epsilon_{\mathrm{eff}}^{\prime} \epsilon}, \\
\epsilon_{\mathrm{eff}} & =\epsilon_{\mathrm{eff}}^{\prime}+i \epsilon_{\mathrm{eff}}^{\prime \prime}=\epsilon \frac{1+2 f y}{1-f y}, \quad y=\frac{\epsilon_{s}-\epsilon}{\epsilon_{s}+2 \epsilon} .
\end{aligned}
$$

This discrepancy may seem surprising, since the MG takes into account the influence of nearby particles. However, the MG uses a low-frequency approximation and does not vary as a function of frequency. We found that as the fractional volume increases, we needed to decrease $k a$ to produce absorption rates that agree with the MG. For example, at 20\% fractional volume the MG predicts an absorption coefficient of $\kappa_{a} / k=0.7 \times 10^{-3}$. For $k a=0.2$ Monte Carlo simulations give an absorption rate of $\kappa_{a} / k=0.8 \times 10^{-3}$. If $k a$ is decreased to $k a=0.02$ (i.e., lower frequency), our calculated values agree with those of the MG. These results suggest that, for densely packed particles, the threshold for low-frequency approximations depends on fractional volume.

Monte Carlo simulations were also run for spheres with a stickiness of $\tau=0.2$ and $\epsilon_{s}=(3.2+i 0.01) \epsilon_{0}$. The resulting absorption rates showed the effect of absorption enhancement but did not differ significantly from those in the nonsticky case. This indicates that the absorption is not affected by clustering or sphere placement.

The absorption rates in Fig. 7 were calculated from the electric dipole only. We also calculated the absorption resulting from the magnetic-field contribution and found that it was several orders of magnitude smaller than that appearing as a result of the electric dipole. In Fig. 8 the absorption rates that are due to the electric and magnetic dipoles are shown as a function of $k a$. The contribution of the magnetic dipole increases more rapidly as a function of $k a$ than does the contribution of the electric dipole, but its magnitude is still negligible in comparison with the small $k a$ considered (note the different scales). However, the rapid growth of this term indicates that the effect of the magnetic field increases in higher-frequency regimes.

\section{CONCLUSIONS}

In this paper we have presented scattering and absorption coefficients from Monte Carlo simulations of densely packed dielectric, absorbing spheres. The spheres were deposited into a test volume in a random fashion, both with and without surface adhesion. Scattering and absorption were calculated by a numerically exact iterative formulation of Maxwell multiple-scattering equations, in which the contributions of the electric and magnetic dipoles were considered.

Results of these simulations show a clear dependence on the initial placement of the spheres. When interparticle adhesive forces cause clustering of the spheres, the scattering increases because of the effectively larger particle that the clusters represent. This is in agreement with previous research on single fractal aggregations, which also found an increase in scattering. Both the QCA-CP and the Monte Carlo simulations predict this increase in scattering for sticky spheres relative to that in the nonsticky case, but the magnitude of the increase is overestimated by the QCA-CP. The increase is less pronounced at higher fractional volumes, since the declining freedom of sphere placement curtails the clustering behavior.

For nonsticky particles the scattering predicted by the QCA and the QCA-CP agrees well with the results of the Monte Carlo simulations. At higher fractional volumes the QCA and the QCA-CP predict slightly lower extinction rates than those from the Monte Carlo simulations, perhaps because of the low-frequency approximations used in the solution for the QCA and the QCA-CP. The validity of the low-frequency approximation depends on $k a$ and on the fractional volume. For a given $k a$ there is a threshold fractional volume above which the low-frequency assumption no longer holds. The value of the threshold decreases as $k a$ increases.

For both sticky and nonsticky spheres the absorption coefficient for an ensemble of absorbing spheres was larger than that predicted by assuming independent absorption. This is due to a modulation of each sphere's local exciting field as a result of the neighboring particles. In essence, the high local fields aid the ability of each sphere to absorb incident energy. The validity of the independent-absorption assumption again depends on $k a$ and the fractional volume, as observed for the validity of the low-frequency approximations used in the QCA-CP. Results from a low-frequency approximation begin to disagree with the Monte Carlo results when fractional volumes greater than a threshold value are considered. The value of the threshold decreases with increasing $k a$.

\section{ACKNOWLEDGMENTS}

This work was supported by the U.S. Office of Naval Research (grant N00014-89-J-313) and the U.S. Army Research Office (grant DAAL03-91-G-0229).

\section{REFERENCES}

1. L. Tsang and J. A. Kong, "Scattering of electromagnetic waves from a half space of densely distributed dielectric scatterers," Radio Sci. 18, 1260-1272 (1983).

2. K. H. Ding, L. M. Zurk, and L. Tsang, "Pair distribution functions and attenuation rates for sticky particles in dense media," J. Electromagn. Waves Appl. 8, 1585-1604 (1994).

3. A. Ishimaru, Wave Propagation and Scattering in Random Media (Academic, New York, 1978), Vols. 1 and 2.

4. A. Ishimaru and Y. Kuga, "Attenuation constant of a coherent field in a dense distribution of particles," J. Opt. Soc. Am. 72, 1317-1320 (1982). 
5. Y. Kuga and A. Ishimaru, "Retroreflectance from a dense distribution of spherical particles," J. Opt. Soc. Am. A 1, 831-835 (1984).

6. G. Koh, "Experimental study of electromagnetic wave propagation in dense random media," Waves Random Media 2, 39-48 (1992).

7. V. Twersky, "Coherent scalar field in pair-correlated random distributions of aligned scatterers," J. Math. Phys. 18, $2468-2486$ (1977).

8. V. K. Varadan, V. N. Bringi, V. V. Varadan, and A. Ishimaru, "Multiple scattering theory for waves in discrete random media and comparison with experiments," Radio Sci. 18, 321-327 (1983).

9. L. Tsang, C. Mandt, and K. H. Ding, "Monte Carlo simulations of the extinction rate of dense media with randomly distributed dielectric spheres based on solution of Maxwell's equations," Opt. Lett. 17, 314-316 (1992)

10. C. E. Mandt, Y. Kuga, L. Tsang, and A. Ishimaru, "Microwave propagation and scattering in a dense distribution of nontenuous spheres: experiment and theory," Waves Random Media 2, 225-234 (1992).

11. Y. M. Wang and W. C. Chew, "Electromagnetic scattering from a cluster of spheres," in IEEE Antennas and Propagation Society International Symposium (Institute of Electrical and Electronics Engineers, New York, 1992), Vol. 3, pp. 929-932.

12. L. Roth, "Effective medium approximation for liquid metals," Phys. Rev. B, 9, 2476-2484 (1974).

13. V. A. Davis and L. Schwartz, "Electromagnetic propagation in close-packed disordered suspensions," Phys. Rev. B 31, 5155-5165 (1985).

14. L. Tsang and J. A. Kong, "Multiple scattering of electromagnetic waves by random distribution of discrete scatterers with coherent potential and quantum mechanical formulism,” J. Appl. Phys. 51, 3465-3485 (1980).

15. L. Tsang and J. A. Kong, "Effective propagation constants for coherent electromagnetic wave propagation in media embedded with dielectric scatterers," J. Appl. Phys. 53, 7162-7173 (1982).
16. L. Tsang, J. A. Kong, and R. T. Shin, Theory of Microwave Remote Sensing (Wiley-Interscience, New York, 1985).

17. R. West, D. Gibbs, L. Tsang, and A. K. Fung, "Comparison of optical scattering experiments and the quasi-crystalline approximation for dense media," J. Opt. Soc. Am. A 11, 1854-1858 (1994)

18. R. J. Baxter, "Percus-Yevick equation for hard spheres with surface adhesion,” J. Chem. Phys. 49, 2770-2773 (1968).

19. J. P. Hansen and I. R. McDonald, Theory of Simple Liquids (Academic, New York, 1986)

20. J. K. Percus and G. J. Yevick, "Analysis of classical statistical mechanics by means of collective coordinates," Phys. Rev, 110, 1-13 (1958).

21. N. A. Seaton and E. D. Glandt, "Monte Carlo simulation of adhesive spheres," J. Chem. Phys. 87, 1785-1790 (1977).

22. W. G. T. Kranendonk and D. Frenkel, "Simulation of the adhesive-hard-sphere model," Mol. Phys. 64, 403-424 (1988).

23. T. Vicsek, Fractal Growth Phenomena (World Scientific, Teaneck, New Jersey, 1992).

24. B. B. Mandelbrot, The Fractal Geometry of Nature (Freeman, New York, 1983).

25. H. Y. Chen and M. F. Iskander, "Light scattering and absorption by fractal agglomerates and coagulations of smoke aerosols," J. Mod. Opt. 37, 171-181 (1990).

26. V. M. Shalaev, R. Botet, and R. Jullien, "Resonant light scattering by fractal clusters," Phys. Rev. B 44, 12216-12225 (1991).

27. H. C. van de Hulst, Light Scattering by Small Particles (Dover, New York, 1957)

28. B. Peterson and S. Ström, " $T$ matrix for electromagnetic scattering from an arbitrary number of scatterers and representations of E(3)*," Phys. Rev. D 8, 3661-3678 (1973).

29. L. Tsang and J. A. Kong, "Multiple scattering of acoustic waves by random distributions of discrete scatterers with the use of quasicrystalline-coherent potential approximation," J. Appl. Phys. 52, 5448-5458 (1981).

30. J. C. Maxwell-Garnett, "Colours in metal glasses and in metal films," Trans. R. Soc. London CCIII, 385-420 (1904). 